\title{
Supersensitive Viral Load Assay in Predicting CD4-Guided Treatment Failure
}

\author{
Simone Langford ${ }^{\mathrm{a},}$, Angele Gayet-Ageron $^{\mathrm{b},}$, Chris Duncombe $^{\mathrm{a}}$, Thidarat Jupimai ${ }^{\mathrm{a}}$, \\ Apicha Mahanontharit ${ }^{\mathrm{a}}$, Sasisopin Kiertiburanakul ${ }^{\mathrm{c}}$, Warangkana Munsakul ${ }^{\mathrm{d}}$, \\ Kiat Ruxrungtham $^{\mathrm{a}, \mathrm{e}}$, Bernard Hirschel ${ }^{\mathrm{b}}$, Jintanat Ananworanich ${ }^{*}$,a, \\ and the Staccato Study Group
}

${ }^{a}$ The HIV Netherlands Australia Thailand Research Collaboration (HIV-NAT), Bangkok, Thailand ${ }^{b}$ HIV Unit, Infectious Disease Department, Geneva University Hospital, Geneva, Switzerland ${ }^{c}$ Faculty of Medicine, Ramathibodi Hospital, Mahidol University, Bangkok, Thailand

${ }^{d}$ Bangkok Metropolitan Administration Medical College and Vajira Hospital, Bangkok, Thailand

${ }^{e}$ Faculty of Medicine, Chulalongkorn University, Bangkok, Thailand

${ }^{f}$ South East Asia Research Collaboration with Hawaii, Bangkok, Thailand

\begin{abstract}
In HIV patients who discontinue highly active antiretroviral therapy (HAART), the degree of HIV RNA suppression at the time of treatment interruption may predict success of re-treatment after the interruption (STI). A casecontrol substudy of the Staccato trial in Thailand included CD4-guided STI subjects with HIV RNA > $50 \mathrm{copies} / \mathrm{ml}$ (virological failure cases, $\mathrm{n}=11$ ) and HIV RNA $<50$ copies/ml (controls, $\mathrm{n}=22$ ) after 12-24 weeks of HAART re-treatment following a median of 2 STI cycles. Controls were matched for age, gender and pre-ART CD4 count. HIV RNA with 5 copies/ml detection limit was determined on pre-virological failure samples. HIV RNA increased in cases compared to controls with each successive STI cycle (p-trend across time-points 0.004). The last HIV RNA below $50 \mathrm{copies} / \mathrm{ml}$ was significantly higher among cases compared to controls ( $\mathrm{p}=.004)$. Measuring HIV RNA below 50 copies/ml may be useful in predicting virological failure to STI.
\end{abstract}

\section{INTRODUCTION}

HIV-RNA quantification is a sensitive indicator of the effectiveness of highly active antiretroviral therapy (HAART). HIV RNA measurements 2-6 months after treatment initiation correlate with long-term virological outcomes $[1,2]$. Successful HAART is generally defined as HIV RNA suppression to below 50 copies $/ \mathrm{ml}$ although low level replication continues even when HIV RNA is undetectable by conventional assays [3-5].

Staccato investigated CD4-guided structured treatment interruption (STI) of HAART and found that the rate of virological failure was low (2\%) and similar to those who took HAART continuously [6]. While most STI patients in our study achieved HIV RNA suppression below 50 copies $/ \mathrm{ml}$ following HAART re-treatment, it is possible that slow rises in HIV RNA with successive STI cycles occur and result in subsequent virological failure in some patients. In this substudy, we investigated the value of a modified version of the Roche AMPLICOR Monitor 1.5 protocol with a limit of

\footnotetext{
*Address correspondence to this author at the HIV Netherlands Australia Thailand Research Collaboration (HIV-NAT), 104 Rajdumri Road, Pathumwan, Bangkok 10330, Thailand; Tel: +66-2-255-7335, Ext. 129; Fax: +66-2-252-5779; E-mail: jintanat.a@ @ivnat.org

${ }^{\S}$ These Authors Contributed Equally
}

detection of 5 copies $/ \mathrm{ml}$, in predicting virological failure after STI. We hypothesized that compared to patients with HIV RNA $<5$ copies/ml, those with HIV RNA between 5-49 copies/ml following HAART re-treatment were more likely to have virological failure after CD4-guided STI.

\section{MATERIALS AND METHODS}

\section{Study Population}

This was a sub-study of the Staccato Trial which was performed in Thailand only $(n=379,77 \%$ of the total Staccato population). The study design is shown in Fig. (1). In brief, Staccato enrolled HAART-treated patients with HIV RNA < 50 copies $/ \mathrm{ml}$ and CD4 counts $>350$ cells $/ \mu 1$ and randomized them in a 2:1 fashion to CD4-guided STI, resuming HAART only when CD4 count fell below 350 cells/ $\mu 1$ (STI arm, $n=238$ in Thailand) and continuous treatment ( $n=118$ in Thailand) with their existing HAART regimen. Following a median time of 21.9 months after randomization, all patients received 12 to 24 weeks of HAART and HIV RNA response to re-treatment was determined. The HAART regimen in Thai patients was 2 nucleoside reverse transcriptase inhibitors + ritonavir-boosted saquinavir. Thai patients were antiretroviral-naïve prior to enrollment and received HAART for at least 24 weeks until they fulfilled the randomization criteria. All patients provided written informed consent. The study was approved by the Thai national and local institu- 


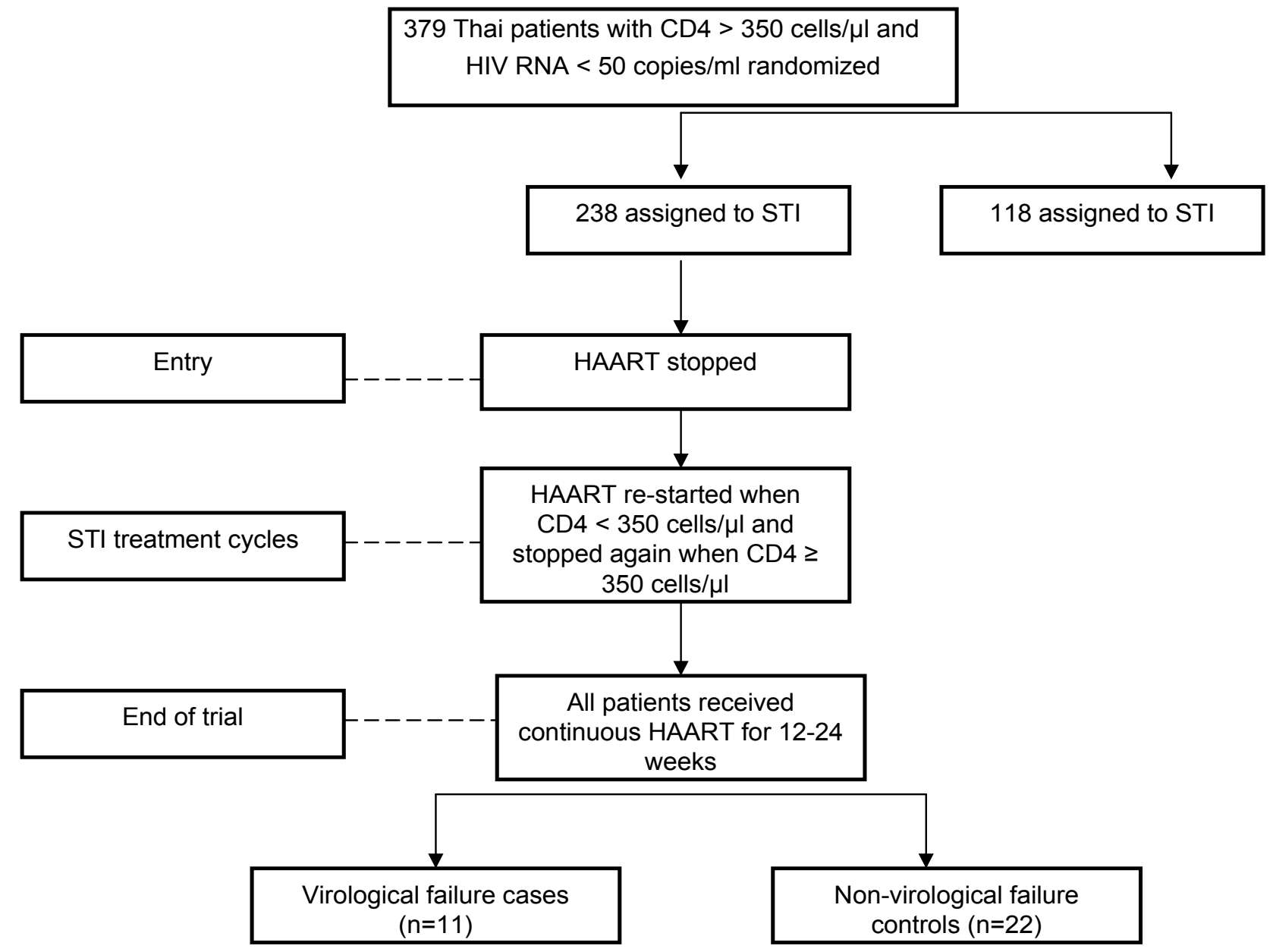

Fig. (1). The study design. HAART (highly active antiretroviral therapy), STI (structured treatment interruption), CT (Continuos Treatment) virological failure cases were defined as patients who had HIV RNA > 50 copies/ml after 24 weeks of HAART re-treatment following CD4guided STI, non-virological failure controls were defined as patients with HIV RNA $\leq 50$ copies/ml after 12-24 weeks of HAART retreatment who were matched with virological failure cases by gender, age and pre-treatment CD4 count.

tional review boards. This study is registered at ClinicalTrials.gov with the identifier NCT00113126.

\section{Definition of Cases and Controls}

Cases: Patients with a virological failure in the STI arm from Staccato: HIV RNA > 50 copies/ml after 24 weeks of HAART re-treatment following CD4-guided STI.

Controls: Patients without virological failure after 12 to 24 weeks of HAART re-treatment following CD4-guided STI: HIV RNA $\leq 50$ copies/ml at 12 or 24 weeks (if HIV RNA at 12 weeks was above 50 and under 500 copies $/ \mathrm{ml}$ ). Two controls were matched per case by gender, age $( \pm 3$ years), pre-treatment CD4 count ( \pm 50 cells).

\section{Study Time-Points}

"Entry" corresponds to the baseline visit, before HAART was stopped for the first time. The first cycle of re-treatment period lasts from randomization to the day when patients achieved suppressed HIV RNA with HAART following their first STI. Similarly, the second cycle of re-treatment lasts from the second treatment stop to the day of HIV RNA suppression with HAART following the second STI. The last HIV RNA below 50 copies/ml described the last time point with HIV RNA below 50 copies/ml prior to the protocolmandated HAART re-treatment period at the end of the trial. The end of the re-treatment period corresponded to the end of Staccato trial, i.e. after 12 to 24 weeks of re-treatment. For cases, this corresponded to the time when they failed to suppress HIV RNA below 50 copies $/ \mathrm{ml}$.

\section{Modified HIV RNA Assay}

The COBAS AMPLICOR Monitor Version 1.5 (Roche, Nutley, USA) was modified to lower the detection limit of 5 copies/ml [3]. In order to increase the initial viral RNA input, plasma samples of $1.1 \mathrm{ml}$ were centrifuged at $50,000 \mathrm{~g}$ at $4^{\circ} \mathrm{C}$ for 90 minutes before RNA extraction. Following removal of the supernatant, the only alterations to the manufacturers' protocol for the Ultrasensitive assay were a decrease in the volume of internal quantitative standard $(6.25 \mu \mathrm{L} v s 25 \mu \mathrm{L})$, a reduction in the volume of specimen diluent $(55 \mu \mathrm{L}$ vs $400 \mu \mathrm{L})$ and extension of the substrate incubation time (15 minutes vs 10 minutes).

\section{Statistical Analysis}

Cases and controls (1:2) were compared at baseline regarding demographical and clinical variables using univari- 
ate conditional logistic regression to compare qualitative variables and Wilcoxon matched-pairs signed-rank test for quantitative variables. The case-control population was also compared to the entire Thai population included in the STI arm of Staccato using unmatched a non parametric test (Mann Whitney) for quantitative data and Fischer's exact test for qualitative data.

P-test for linearity across the study time-points was assessed using ANOVA.

In order to determine whether the last available HIV RNA above 5 copies $/ \mathrm{ml}$ was a predictive factor for virological failure at the end of Staccato, conditional logistic regression was performed using a backward stepwise procedure. Each independent variable with a p-value $<0.25$ at univariate analysis was included in the multivariate model and variables were removed one by one, leaving those with a $\mathrm{p}$ value $<0.10$.

SPSS 15.0 (SPSS Inc. Chicago, Illinois, USA) and STATA 8 Statistical Softwares (StataCorp LP, Texas, USA) were used to perform analyses.

\section{RESULTS}

We included 11 cases and 22 controls. The baseline characteristics of cases and controls are presented in Table $\mathbf{1}$ and compared to those of the Thai population included into Staccato and randomized in the STI arm. Cases and controls were similar, except that cases had significantly more STI cycles than controls $(\mathrm{p}=0.02)$. The case-control population was similar to the entire Thai population included in the STI arm of Staccato, except that Thai patients were significantly older than patients selected in the case-control study $(\mathrm{p}=0.04)$.

Table 2 shows the median HIV RNA values for the cases and controls, with their interquartile range (IQR), at each STI re-treatment cycle. In cases, the median (IQR) duration of the first and the second STI cycles weres 24 and 36 weeks respectively. In controls, the median duration of the first cycle was 12 weeks, and 24 weeks for the second cycle. Using modified HIV RNA HIV RNA assay with a detection limit of 5 copies $/ \mathrm{ml}$, we did not find any difference between cases and controls on $\log 10$ HIV RNA at entry $(p=0.24)$. After the first cycle of re-treatment, there was a trend to a higher HIV RNA in cases compared to controls $(p=0.06)$. After the second cycle of re-treatment, there was no significant difference cases and controls regarding the median HIV RNA ( $p=0.32)$. When we used the last available HIV RNA below 50 copies $/ \mathrm{ml}$ for cases and controls, we found that cases had a significantly higher median HIV RNA than the controls $(p=0.004)$. Variations in trend across study timepoints showed a significant increase in $\log 10$ HIV RNA (ptrend $=0.004$ ) only due to a significant increase in cases ( $\mathrm{p}$ trend $<0.001$ vs 0.55 in controls).

By univariate and multivariate conditional logistic regression model, there was a trend for a higher probability of final virological failure when the last available HIV RNA was between 5-49 copies/ml compared to HIV RNA below 5 copies $/ \mathrm{ml}(\mathrm{OR} 7.77,95 \%$ CI 0.89-67.5, $\mathrm{p}=0.06)$. There was no significant association between the risk to have HIV RNA between 5-49 copies $/ \mathrm{ml}$ and risk factor, CDC classification and pre-HAART CD4.

\section{DISCUSSION}

With each successive treatment interruption, the differences in median HIV RNA between virological failure cases and controls increased, reaching statistical significance with the last available HIV RNA prior to the protocol-mandated HAART re-treatment at the end of the trial. This finding implies that over the course of multiple interruptions there is a trend towards higher residual viremia amongst those who ultimately experienced treatment failure than in those who maintained low HIV RNA.

Our result is similar to the ISS PART study which showed increased residual HIV viremia between 2.5 to 50 copies $/ \mathrm{ml}$ in virological failure cases after two years of a step-wise fixed time strategy with five STIs of one, one, 2, 2

Table 1. Characteristics of STI Participants in this Substudy and in the Whole Staccato Thai Cohort

\begin{tabular}{|c|c|c|c|c|c|}
\hline Characteristics & $\begin{array}{c}\text { Cases } \\
(n=11)\end{array}$ & $\begin{array}{c}\text { Controls } \\
(n=22)\end{array}$ & p-Value & $\begin{array}{l}\text { Staccato STI Thai Cohort } \\
(\mathbf{n}=\mathbf{2 3 8})\end{array}$ & p-Value \\
\hline Median age in years (IQR) & $33.5(31-38)$ & $32.5(29-36.5)$ & 0.52 & $34.5(29.9-40.0)$ & 0.04 \\
\hline Gender (\% male) & $11(100)$ & $22(100)$ & 0.99 & $105(44.1)$ & 0.33 \\
\hline Mode of transmission (\%) & & & 0.43 & & 0.99 \\
\hline MSM & $2(18.2)$ & $2(9.1)$ & & $27(11.3)$ & \\
\hline CDC stage $(\%)$ & & & & & 0.99 \\
\hline CDC A & $6(54.6)$ & $15(68.2)$ & - & $146(61.3)$ & \\
\hline $\mathrm{CDC} B$ & $5(45.4)$ & $6(27.3)$ & 0.19 & $79(33.2)$ & \\
\hline $\mathrm{CDC} \mathrm{C}$ & 0 & $1(4.5)$ & 0.99 & $13(5.5)$ & \\
\hline Median pre-STI CD4 cells/ $\mu \mathrm{L}$ (IQR) & $463(398-570)$ & $433(369-570)$ & 0.18 & $463(399-553)$ & 0.42 \\
\hline
\end{tabular}

IQR: interquartile range, STI: structured treatment interruption, MSM: Men who have sex with men, ARV: Antiretroviral therapy. 
Table 2. Median HIV RNA in Copies/ml (Interquartile Range) Between Cases and Controls After HAART Re-Treatment

\begin{tabular}{|l|c|c|c|c|c|}
\hline \multirow{2}{*}{\multicolumn{2}{c|}{ Time Points }} & \multicolumn{2}{c|}{ Cases } & \multicolumn{2}{c|}{ Controls } \\
\cline { 2 - 6 } & Median & IQR & \multirow{2}{*}{ Median } & IQR \\
\hline \hline Entry & 5 & $5-17$ & 5 & $5-10$ & 0.24 \\
\hline $1^{\text {st }}$ HAART re-treatment & 15 & $11-24$ & 5 & $5-15$ & 0.06 \\
\hline $2^{\text {nd }}$ HAART re-treatment & 29 & $28-30$ & 5 & $5-10$ & 0.32 \\
\hline Last available HIV RNA < 50 copies/ml & 17 & $8-28$ & 5 & $5-11$ & 0.004 \\
\hline Last HAART re-treatment at the end of the trial & 190 & $98-470$ & 5 & $5-11$ & $<0.001$ \\
\hline
\end{tabular}

*We provided p-value from Wilcoxon signed-rank test on values expressed in log 10 copies $/ \mathrm{ml}$

and 3 months' duration, each followed by 3 months of HAART [7]. The ISS PART subjects were different to the Staccato subjects. They were mostly antiretroviral-pre treated and had archived resistance mutations. The Staccato Thai subjects were HAART-naïve and likely had no archived mutations [6]. This illustrates that the finding of increased residual viremia after STI may apply to both those with and without pre-existing risk for virological failure.

The treatment failures seen after STI have mostly been attributed to the replication of archived resistant quasispecies that existed in proviral DNA prior to STI. Selection of new mutations have been reported in some studies especially those that use antiretrovirals with low resistance threshold such as lamivudine and non-nucleoside reverse transcriptase inhibitors [8, 9]. The short duration of HAART-retreatment after each STI cycle may be inadequate to control HIV viral replication in viral reservoirs and compartments.

Currently, the goal of HAART is to maintain HIV RNA below 50 copies $/ \mathrm{ml}$ [2]. Studies have shown continued viral replication at levels below 50 copies/ml despite HAART (3, 4]. Palmer et al. used an HIV-1 RNA assay with single-copy sensitivity to show that $77 \%$ of 40 HAART-treated patients with HIV RNA below 50 copies/ml for at least 2 years had detectable low-level viremia, and that all patients had at least one sample with detectable viremia [4]. The significance of this finding is unclear. It may explain the viral blips that occurs frequently but do not appear to lead to virological failure with both protease inhibitor and non-nucleoside reverse transcriptase treatments [10-12]. Continued viremia at varying HIV RNA levels albeit below 50 copies/ml may have different effects on the HIV reservoirs. Persistence of viral reservoir particularly the latently infected CD4+ T cells has been a major obstacle to HIV eradication $[13,14]$. With new HAART becoming more effective, it is possible that in the future suppressing HIV to the lowest possible level using HIV RNA monitoring assay such as that in our study may be desired in an attempt to limit the chance of HIV rebound and the size of HIV reservoir.

Our study is limited by its small sample size and the highly selective nature of the case-control matching which may limit the generalizability of the results to other populations. Nevertheless, by utilizing a modified HIV RNA method, we demonstrated that a higher residual viremia between 5 and 50copies/ml after HAART re-treatment may predict those who would ultimately experienced treatment failure from STI.

\section{CONFLICT OF INTEREST}

J.A. has received travel grants and speakers' honoraria from Roche. K.R. has received travel grants, consultancy fees and speakers' honoraria from Roche, Abbott and Bristol-Myer-Squibb. B.H. has received travel grants and speakers' honoraria from Roche, Abbott and Gilead. All the others have not accepted financial contribution, which may affect the conclusion of this article. No authors own stocks from companies involved in this work.

\section{ACKNOWLEDGEMENTS}

The Staccato study was funded by grants from Swiss National Science Foundation through the Swiss HIV Cohort Study, the Wilsdorf, Sidaide, and de Brocard Foundations, Geneva, from the Department of Social Affairs and Economics, Geneva, and Roche. Roche provided HIV RNA kits for this substudy. Roche, Abbott and Gilead donated antiretrovirals used in Staccato. Bristol-Myer-Squibb sold antiretrovirals at a reduced price to Staccato.

\section{The Staccato Thailand Study Group}

Protocol Advisors: Praphan Phanuphak (The HIV Netherlands Australia Thailand Research Collaboration, Bangkok, Thailand), David A. Cooper (The National Centre in HIV Epidemiology and Clinical Research, Sydney, Australia).

Investigators: Kiat Ruxrungtham, Jintanat Ananworanich and Reto Nuesch (HIV-NAT, Bangkok), Ploenchan Chetchotisakd (Khon Kaen University, Khon Kaen), Wisit Prasithsirikul (Bamrasnaradura Institute, Nonthaburi), Sasisopin Kiertiburanakul (Faculty of Medicine, Ramathibodi Hospital, Mahidol University, Bangkok), Warangkana Munsakul (Bangkok Metropolitan Administration Medical College and Vajira Hospital, Bangkok), Phitsanu Raksakulkarn and Wirat Klinbuayam (Sanpatong Hospital, Chiang Mai, Thailand), Somboon Tansuphasawadikul (Buddhachinnaraj Hospital, Phitsanulok, Thailand).

Study Nurses, Monitors and Laboratory Technicians: Bangkok: Sukontha Saenawat, Saijai Wicharuk, Siriporn Nonenoy, Natnipa Wannachai, Sineenart Chautrakarn, Theshinee Chuenyam, Thantip Nuchapong, Apicha Mahanontharit, Jongkol Sankote, Wiphawee Kiatatchasai, Bucha Piyavong, Vantanit Pairoj, Napawan Seekaow, Wipawan Karakate. Nonthaburi: Suchittra Putthawong, Wattana Sanchiem, Sirirat Liganonsakul, Pongpan Boonchoo, Malee Suannum. Khon Kaen: Parichat Bunyaprawit, Ratthanant 
Kaewmarg. Chiang Mai: Yaowaluk Penglimoon. Phitsanulok: Sopha Khongsawad, Switzerland: Michelle Le Braz.

\section{REFERENCES}

[1] van Leth F, Andrews S, Grinsztejn B, et al. The effect of baseline CD4 cell count and HIV-1 viral load on the efficacy and safety of nevirapine or efavirenz-based first-line HAART. AIDS 2005; 19: 463-71.

[2] Guidelines for the use of antiretroviral agents in HIV-infected adults and adolescents. U.S. Department of Health and Human Services (DHHS), www.aidsinfo.nih.gov, January 29, 2008.

[3] Yerly S, Perneger TV, Vora S, Hirschel B, Perrin L. Decay of cellassociated HIV-1 DNA correlates with residual replication in patients treated during acute HIV-1 infection. AIDS 2000; 14: 280512.

[4] Palmer S, Maldarelli F, Wiegand A, et al. Low-level viremia persists for at least 7 years in patients on suppressive antiretroviral therapy. Proc Natl Acad Sci USA 2008; 105: 3879-84.

[5] Palmisano L, Giuliano M, Nicastri E, et al. Residual viraemia in subjects with chronic HIV infection and viral load < 50 copies $/ \mathrm{ml}$ : the impact of highly active antiretroviral therapy. AIDS 2005; 19: 1843-7.

[6] Ananworanich J, Gayet-Ageron A, Le Braz M, et al. CD4-guided scheduled treatment interruptions compared with continuous therapy for patients infected with HIV-1: results of the Staccato randomised trial. Lancet 2006; 368: 459-65.

[7] Palmisano L, Giugliano M, Bucciardini R, et al., Eds. Residual viremia, but not HIV DNA load, increases in subjects undergoing repeated treatment interruptions: a substudy of ISSPART Clinical Trial [Abstract 515]. 14th Conference on Retroviruses and Opportunistic Infections; February 15-28, 2007; Los Angeles.

[8] Metzner KJ, Bonhoeffer S, Fischer M, et al. Emergence of minor populations of human immunodeficiency virus type 1 carrying the M184V and L90M mutations in subjects undergoing structured treatment interruptions. J Infect Dis 2003; 188: 1433-43.

[9] Dybul M, Nies-Kraske E, Daucher M, et al. Long-cycle structured intermittent $v s$ continuous highly active antiretroviral therapy for the treatment of chronic infection with human immunodeficiency virus: effects on drug toxicity and on immunologic and virologic parameters. J Infect Dis 2003 ; 188: 388-96.

[10] Nettles RE, Kieffer TL, Kwon P, et al. Intermittent HIV-1 viremia (Blips) and drug resistance in patients receiving HAART. JAMA 2005; 293: 817-29.

[11] Havlir DV, Bassett R, Levitan D, et al. Prevalence and predictive value of intermittent viremia with combination hiv therapy. JAMA 2001; 286: 171-9.

[12] Martinez V, Marcelin AG, Morini JP, et al. HIV-1 intermittent viraemia in patients treated by non-nucleoside reverse transcriptase inhibitor-based regimen. AIDS 2005; 19: 1065-9.

[13] Chun TW, Justement JS, Moir S, et al. Decay of the HIV reservoir in patients receiving antiretroviral therapy for extended periods: implications for eradication of virus. J Infect Dis 2007; 195: 17624.

[14] Re MC, Vitone F, Bon I, Schiavone P, Gibellini D. Meaning of DNA detection during the follow-up of HIV-1 infected patients: a brief review. New Microbiol 2006; 29: 81-8.

(C) Langford et al.; Licensee Bentham Open.

This is an open access article licensed under the terms of the Creative Commons Attribution Non-Commercial License (http://creativecommons.org/licenses/by$\mathrm{nc} / 3.0 /$ ) which permits unrestricted, non-commercial use, distribution and reproduction in any medium, provided the work is properly cited. 